\title{
Salivary Biomarker Interleukin-8 Levels in Naswar Users and Non-users
}

\author{
Kanwal Sohail1, Khalid Mahmood Siddiqi2, Muhammad Zeeshan Baig2, Haafsa Arshad Sahibzada1 and Shahida Maqbool1 \\ Department of Oral Medicinel / Oral and Maxillofacial Surgery², Islamabad Medical and Dental College, Islamabad, Pakistan
}

\begin{abstract}
Salivary biomarkers can be used as diagnostic and predictive aids in early detection of oral cancer or potentially malignant disorders. A cross-sectional comparative study was conducted over a period of one year from August 2016 to August 2017 in a multicentre setting in Islamabad. A total of 60 patients were recruited and divided into two equal groups of naswar users and non-users. Un-stimulated saliva samples were collected and analysed by using an enzyme-linked immunosorbent assay (ELISA) technique. Data was entered in SPSS version 22.0. The results were then analysed by using independent t-test. Statistically significant difference was found regarding the levels of salivary IL-8 between the naswar users and non-users $(p<0.001)$. The levels of salivary IL-8 in non-users were $33.39 \pm 22.44 \mathrm{pg} / \mathrm{ml}$, while the increased levels of salivary IL-8 in naswar users were found to be $173.48 \pm 46.52 \mathrm{pg} / \mathrm{ml}$.
\end{abstract}

Key Words: Interleukin-8, Smokeless tobacco, Saliva, Proteomics, Early diagnosis.

How to cite this article: Sohail K, Siddiqi KM, Baig MZ, Sahibzada HA, Maqbool S. Salivary biomarker interleukin-8 levels in naswar users and non-users. J Coll Physicians Surg Pak 2020; 30(1):99-101.

Oral cancer is the sixth most common cancer worldwide. The one year data collection regarding the treatment of head and neck cancers in Pakistan showed that the area of rural Sindh comprises of greater frequency of head and neck cancer, which is around $22.6 \%$, than Punjab having $13.4 \%$, Islamabad $13.1 \%$, Balochistan $11.4 \%$ and Khyber Pukhtoonkhwa 8.6\%. A five-year survival rate for oral and pharyngeal cancer is about $50 \%$ for both genders. ${ }^{1}$ The most prevalent form of oral carcinoma in South and South-East Asian region is squamous cell carcinoma, which is around $95 \%$. This occurs because of the excessive use of smokeless tobacco (SLT) including naswar, betel nut, pan, areca nut, cigarette smoking, smokeless tobacco, and gutka. More than $90 \%$ of the smokeless tobacco consumed worldwide is limited to South-East Asia, which makes an estimation of about 100 million people using SLT in India and Pakistan only. ${ }^{2}$ A meta-analysis in Cochrane review regarding the screening for oral cancer claims that there is a lack of strong evidence of any reliable screening procedure for reducing the mortality rate in screened groups. 3 Therefore, early detection of oral malignancy greatly ameliorates the chances of survival.

Saliva has a number of benefits of a noninvasive procedure of sample collection, smaller sample requirement, good cooperation of patients being a stress, free and painless procedure, easy transportation and storage, higher

Correspondence to: Dr. Kanwal Sohail, Department of Oral Medicine, Islamabad Medical and Dental College, Islamabad, Pakistan E-mail: drkanwalsa@gmail.com

Received: October 29, 2018; Revised: March 18, 2019;

Accepted: July 04, 2019 sensitivity and specificity, and a source of diverse constituents. 4

Cytokines like interleukins (IL) e.g. interleukin-8 (IL-8), interleukin-6 (IL-6), interleukin-1 beta (IL-1 $\beta$ ), interleukin-1 alpha (IL-1 $\alpha$ ) and the tumor necrosis factor alpha (TNF- $\alpha$ ) increase cell growth, invading and disrupting the mechanisms of tumor suppression and host immune status, resulting in development of cancer. Several proinflammatory cytokines like IL-1 $\alpha$, IL-6, IL-8, TNF- $\alpha$ and granulocyte-macrophage colony-stimulating factor (GM-CSF) are detected in increased amounts in the tissues and serum of head and neck squamous cell carcinoma patients. 5 These molecular biomarkers, especially salivary IL-8, may become a potential useful screening tool for oral cancer in wide application as it plays an important role in many physiologic and pathologic activities going on in the body.

The aim of this study was to evaluate any change in the levels of interleukin-8 cytokine in relation to naswar and to predict the usefulness of this particular biomarker in the early detection of oral cancer in high risk groups; and hence, reducing the risk of development of cancer and improving the survival rate of the patient.

A comparative cross-sectional study approved by the Institutional Review Board (IRB No: F.2-11/SZAMBU/ AS\&RB/2015/) was conducted from August 2016 to August 2017 in a multicentre setting including Islamabad Dental Hospital and Akbar Niazi Teaching Hospital (ANTH). The total sample size was 60 , with 30 subjects in the control group: non-users, and the study group: naswar users; inducting using non-probability, convenience sampling technique. The study included 
healthy individuals between the ages of 20 to 60 years using naswar for the past one year or more in the study group. Individuals having same age and gender as the study group were recruited in control group. Individuals having previous history of dermal, rheumatic, cancerous, diabetes, cardiovascular, dysplastic or any acute viral condition, immunodeficiency, periodontal disease (BPE score 3 and 4 ), insufficient salivary flow rate, history of consumption of alcohol or tobacco (any form: dry snuff, paan, smoking) and any previous or ongoing radiotherapy and chemotherapy were excluded. Written consent was taken from all the study participants. Clinical examination and history taking was undertaken to rule out any oral mucosal changes or any systemic illness or any other confounding factor. Basic periodontal examination was also done to rule out any periodontal disease.

A morning sample of unstimulated whole saliva was collected by saliva collecting device according to manufacturer's instructions (PureoSAL ${ }^{\mathrm{TM}}$, Oasis Diagnostics ${ }^{\circledR}$ Corporation, Vancouver, USA). The Eppendorf tube was placed in the transferring medium, which is an insulated container maintaining its temperature between $2-8^{\circ} \mathrm{C}$ and then taken to the respective storage freezer within 1-2 hours. The freezer temperature was maintained at $-80^{\circ} \mathrm{C}$ within the Microbiology Laboratory, located in Quaid-e-Azam Hospital, Rawalpindi. On the day of evaluation, the Eppendorf tubes were thawed once only to avoid multiple freeze-thaw cycles, which might compromise the structure of protiens. The temperature was brought between 2 to $8^{\circ} \mathrm{C}$. Enzyme-linked immunosorbent assay (ELISA) procedure was then run on the sample according to the manufacturer's instructions (PicoKine $^{\mathrm{TM}}$, Boster Biological Technology, Pleasanton, USA).

Data from the ELISA test was then entered and analysed in SPSS (Statistical Package for Social Sciences version 22.0). Descriptive analysis was done for the age parameter and IL-8 levels expressed by mean \pm SD. Then independent t-test was applied to compare the levels of salivary IL-8 between the two groups. A p-value equal or less than 0.05 was considered significant at $95 \%$ confidence interval.

Both the naswar users and non-users comprised of males, no female could be allocated in the study because of the regional and cultural practice. A few females found were medically compromised because of which they were excluded. The overall mean age was $27.15 \pm 5.61$ years with the range from 20 to 49 years. The mean age of naswar users was $27.67 \pm 5.69$ years and non-users was $26.63 \pm 5.64$ years. The levels of salivary IL-8 in nonusers were $33.39 \pm 22.44 \mathrm{pg} / \mathrm{ml}$ while the increased levels of salivary IL-8 in naswar users were found to be $173.48 \pm 46.52 \mathrm{pg} / \mathrm{ml} \mathrm{p}<0.001$ (Figure 1).

There are more than 100 potential biomarkers for oral cancer detection reported in the literature. IL-8 plays a

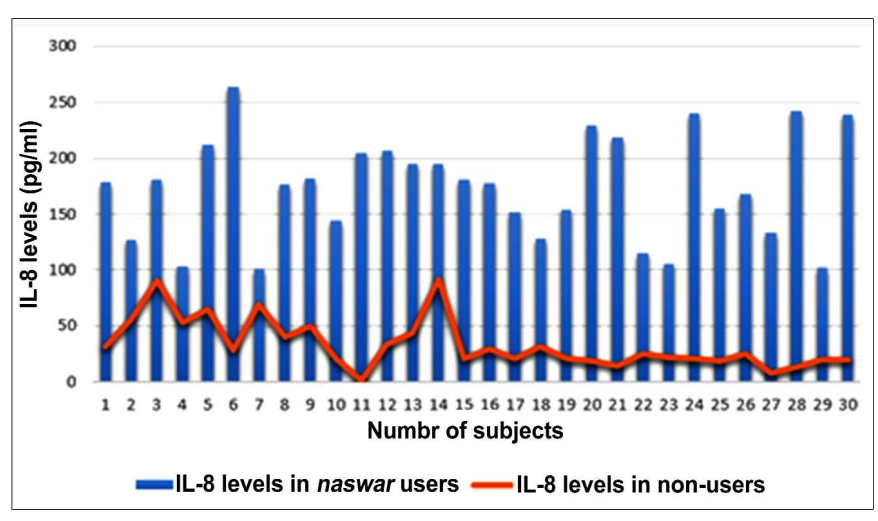

Figure 1: Comparison of salivary IL-8 levels.

significant role in tumor angiogenesis and progression, which makes it a very critical biomarker in regard to oral cancer. Punyani and Sathwane promoted salivary IL-8 as a potential biomarker for oral squamous cell carcinoma (OSCC). ${ }^{6}$ Naswar, being a causative factor of oral cancer, consists of carcinogens like tobacco, nicotine etc. Nicotine plays a role in inducing neutrophils to produce IL-8 through NF-kB pathway. The existence of nicotine in naswar may result in alteration in the salivary levels of cytokines, but very few studies have been conducted to assess any such relationship. Therefore, the present study is targeted to find out any correlation between the risk factors of oral cancer like naswar and the cytokines like IL-8. A local study conducted in Karachi, Pakistan, evaluated the circulatory levels of proinflammatory interleukins like IL-1 $\beta$ and IL-6 in relation to naswar consumption. IL-1 $\beta$ levels were significantly low in naswar users as compared to the non-users while IL-6 showed no difference. ${ }^{7}$

Due to the increased levels of salivary IL-8 in naswar users, one can predict the risk of developing any premalignant or malignant lesion in future. Kaur and Jacobs found that the levels of salivary IL-8 levels in control group were $738.5 \pm 98.5 \mathrm{pg} / \mathrm{ml}$, whereas in leukoplakia with severe dysplasia were $919.87 \pm 107.65$ $\mathrm{pg} / \mathrm{ml}$, in lichen planus with severe dysplasia were $1,116.28 \pm 118.17 \mathrm{pg} / \mathrm{ml}$, and in oral submucous fibrosis with severe dysplasia were $1,437.82 \pm 114.69 \mathrm{pg} / \mathrm{ml}$. Increased concentrations of these cytokines in saliva indicate the potential of these lesions to develop oral cancer. ${ }^{8}$

The use of salivary IL-8 levels in foreseeing the relative risk of a person developing OSCC can be considered. In this regard, the values of salivary IL-8 in naswar users were lower than the values of IL-8 in the precancerous and oral cancer groups, as seen in the literature. It was considerably higher than the non-users to predict the development of any suspicious lesion in high risk population. Longitudinal and case control studies are recommended to enhance the probability of widespread applicability of certain salivary cytokines in the clinical practice. 


\section{PATIENTS' CONSENT:}

Written consents were taken from all the patients.

\section{CONFLICT OF INTEREST:}

Authors declared no conflict of interest.

\section{AUTHORS' CONTRIBUTION:}

$\mathrm{KS}$ : Contributed to the concept, experimental analysis, data interpretation and writing of the manuscript.

KMS: Contributed to the concept, design, supervision of the study and critical revision of the manuscript.

MZB: Contributed to the design and critical revision of the manuscript.

HAS: Contributed to the concept, data collection and writing of the manuscript.

SM: Contributed to the design and critical revision of the manuscript.

\section{REFERENCES}

1. Baumann E, Koller M, Wiltfang J, Wenz HJ, Moller B, Hertrampf K. Challenges of early detection of oral cancer:
Raising awareness as a first step to successful campaigning. Health Educ Res 2016; 31:136-45.

2. Awan $\mathrm{KH}$, Patil S. Association of smokeless tobacco with oral cancer - Evidence from the South Asian studies: A systematic review. J Coll Physicians Surg Pak 2016; 26:775-80.

3. Lambert R, Sauvaget C, de Camargo Cancela M, Sankaranarayanan R. Epidemiology of cancer from the oral cavity and oropharynx. Eur J Gastroenterol Hepatol 2011; 23:633-41.

4. Malathi N, Mythili S, Vasanthi HR. Salivary diagnostics: A brief review. ISRN Den 2014; 2014:1-8.

5. Younus S, Hadi N, Muhammad H. Common salivary bio-chemical markers for early detection of oral squamous cell carcinoma. Pak J Med Dent 2015; 4:39-43.

6. Punyani SR, Sathawane RS. Salivary level of interleukin-8 in oral precancer and oral squamous cell carcinoma. Clin Oral Investig 2013; 17:517-24.

7. Sajid F, Bano S. Pro-inflammatory interleukins and thyroid function in Naswar (dipping tobacco) users: A case control study. BMC Endocrine Disorders 2016; 16:47.

8. Kaur J, Jacobs R. Proinflammatory cytokine levels in oral lichen planus, oral leukoplakia, and oral submucous fibrosis. J Korean Assoc Oral Maxillofac Surg 2015; 41:171-5. 